\title{
Hybrid Design of an Optical Detector for Terrestrial Laser Range Finding
}

\author{
Michael Kufner, Josef Kölbl, Raphael Lukas, Thomas Dekorsy
}

\begin{abstract}
This paper presents the integration of an InGaAs avalanche photo diode (APD) with the first amplification stage, comprised of a low capacitance silicon Nchannel dual-gate MOS-FET and associated passive components, in the APD case. This combined linear mode optical hybrid detector is used in the field of terrestrial laser range finding. In this case, a reduction of input capacitance by $0.7 \mathrm{pF}$ and an improvement of signal-to-noise ratio (SNR) at low background noise conditions by 32 percent when compared to the equivalent discrete detector was measured. When integrated in a laser range finding system at very strong daylight conditions an improvement in SNR by approximately 10 percent was achieved. Furthermore a significant improvement in immunity to electromagnetic interference (EMI) is shown. Finally, when used in an eye-safe mobile laser range finding system, ranging beyond $10 \mathrm{~km}$ with an accuracy of $\pm 2 \mathrm{~m}$ on non-cooperative targets using this hybrid detector is demonstrated.
\end{abstract}

Index Terms_-Photodetectors, Distance Measurement, Hybrid Integrated Circuits

\section{INTRODUCTION}

Laser rangefinders are employed in many civil and military applications to measure the distance to remote objects. Real life examples include applications in golfing to estimate the distance to the hole, in hunting to compensate for bullet trajectories, in naval applications to estimate the distance from ship to land, to estimate target coordinates in assistance with GPS and compass systems, measure direct velocities of incoming or outgoing objects, airborne LiDAR mapping [1], satellite laser ranging [2], construction referencing and many more. There are various different measurement principles like for example triangulation, phase-shift, optical radar or timeof-flight [3]. In this paper we describe the improvement of a time-of-flight laser range finder based on a pulsed laser transmitter. The system architecture is comprised of a laser,

Submit date, etc.

This work was financed by Steiner Optik $\mathrm{GmbH}$, Bayreuth, Germany, supported by Laser Components $\mathrm{GmbH}$, Germany and Laser Components Detector Group, Inc., USA.

Michael Kufner is with the Deggendorf Institute of Technology, Faculty for Applied Natural Sciences and Industrial Engineering, Dieter-GörlitzPlatz 1, 94469 Deggendorf, Germany (e-mail: michael.kufner@thdeg.de). He is currently pursuing a doctoral degree at the University of Stuttgart, Faculty for Aerospace Engineering and Geodesy, Pfaffenwaldring 27, 70569 Stuttgart, Germany.

Josef Kölbl is with the Deggendorf Institute of Technology, Faculty for Applied Natural Sciences and Industrial Engineering, Dieter-GörlitzPlatz 1, 94469 Deggendorf, Germany (e-mail: josef.koelbl@th-deg.de).

Raphael Lukas is with the Deggendorf Institute of Technology, Faculty for Applied Natural Sciences and Industrial Engineering, DieterGörlitz-Platz 1, 94469 Deggendorf, Germany (e-mail: raphael.lukas@thdeg.de).

Thomas Dekorsy is with the German Aerospace Center, Institute of Technical Physics, Pfaffenwaldring 38-40, 70569 Stuttgart, Germany (email: thomas.dekorsy@dlr.de) and the University of Stuttgart, Institute of Aerospace Thermodynamics, Pfaffenwaldring 31, 70569 Stuttgart, Germany. a linear-mode detector with amplifiers, an analog-to-digital converter and a digital processing unit as depicted in Fig. 1. The most important part of the system is the detector unit and its performance in terms of bandwidth and signal-to-noise.

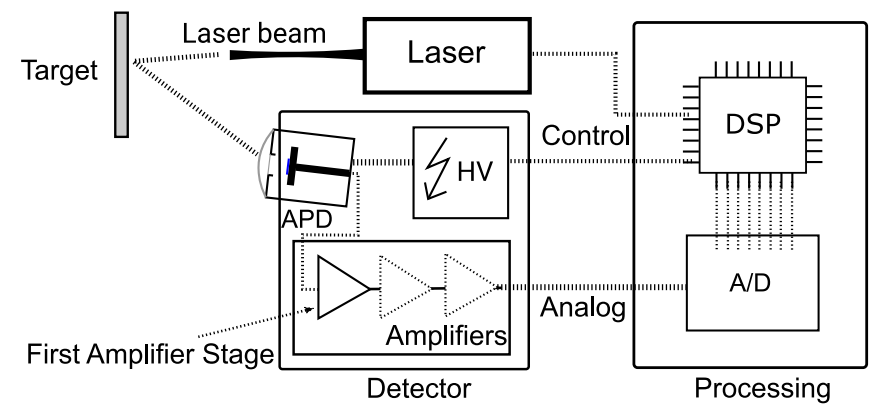

Fig. 1. System overview and key components of a contemporary timeof-flight laser range finder

This paper focuses on the improvement of the first amplification stage, which is the main contributor to the overall performance according to the Friis formula [4]. The first amplification stage in this case, is a discrete voltage amplifier rather than a current amplifier, comprised of a low capacitance and low noise silicon N-channel dual-gate MOS-FET, mitigating the Miller Effect [5], in high impedance configuration followed by an equalizer circuit extending the receiver's bandwidth. It is important to note, that for terrestrial laser range finders, laser eye safety very often is a point of concern and thus limits the permissible laser output power. Also, the detector in this case, is applied to ranging systems, which can range starting at zero up to many kilometers at meter accuracy. Therefore this system only requires relatively low bandwidths compared 
to other recent high-speed application targeted work [6] [7] [8] [9]. The chosen high-impedance detector amplifier circuit topology offers lowest noise level performance and highest sensitivity. The common source amplifier configuration is best when low voltage noise density of the MOS-FET transistor (typ. $1 \mathrm{nV} / \sqrt{\mathrm{Hz}}$ ) is combined with low total input capacitance. The current noise density of the MOSFET is negligible in this case. The following equalizer circuit is required and shall extend the overall receiver bandwidth. It is implemented by a discrete operational amplifier device. However, the popular transimpedance amplifier design will benefit in signal-to-noise ratio and high-speed performance (bandwidths $>50 \mathrm{MHz}$ ), when the total input capacitance exceeds several picofarads typically [10] [11]. The transistor currently used in our system design offers the best compromise of input gate capacitance, flicker corner noise, gate currents, voltage noise as well as transconductance to our knowledge. As a discrete part, it comes in a surface mount package, which introduces parasitic package capacitance. Furthermore the avalanche photo diode (APD) is packaged in a 3-pin TO-46 case. The fact, that these parasitic capacitances are of significant size compared to other input capacitances of the detector, motivates this work to further reduce these parasitics. Improvements of signal-tonoise as well as improvements in electromagnetic interference (EMI) immunity are expected. The signal-to-noise is very important as it defines the maximum ranging capabilities and detection probability of the ranging system.

\section{HYBRID DESIGN}

In order to reduce mainly parasitic capacitances of the ICpackages, the printed circuit board (PCB) tracing and the solder connections, we combined the first amplification stage and the APD into one package.

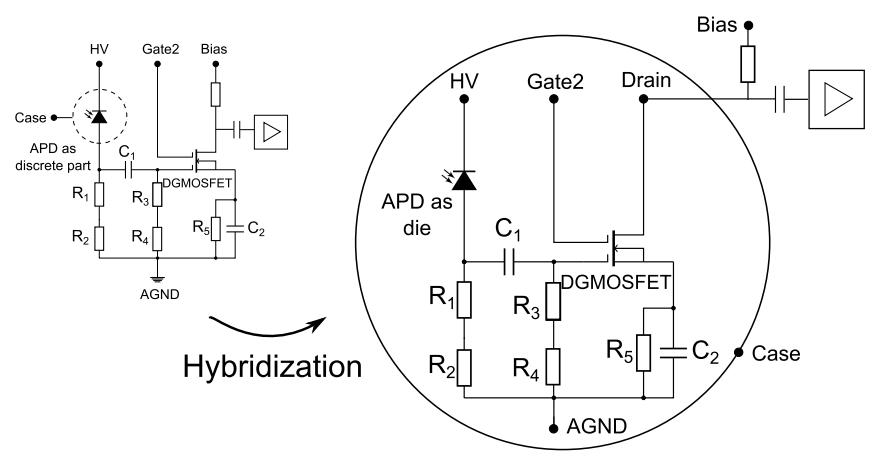

Fig. 2. Integration of discrete frontend amplifier circuit into APD case, left non-hybrid/discrete, right hybrid

As depicted in Fig. 2 the APD in a separate 3-pin TO46 case, the passive components and the dual-gate MOS-FET transistor which were previously mounted on a PCB as a discrete, non-hybrid detector are integrated together into the same case, forming the hybrid detector. This hybridization was performed in cooperation with Steiner Optik GmbH, Germany and Laser Components DG Inc., USA. A batch of 10 samples were manufactured and qualified. In order to optimize performance and reduce the risk in manufacturing, the length of the wirebonds and the number of PCB-trace lines were preemptively minimized and the crossing of wirebonds was explicitly eliminated. Since the device needed to be fit-form compatible with previous designs, the space inside the hybrid detector case is limited. Therefore the non-critical parts $\mathrm{C}_{1}$, $\mathrm{C}_{2}$ and $\mathrm{R}_{5}$ were chosen as discrete, small solderable parts to save space. As especially the capacitive parasitics of $R_{1}+R_{2}$ and $R_{3}+R_{4}$ were of a concern, a thin-film bondable dual resistor was used. Thin-film bondable resistors have smaller stray capacitances when compared to discrete parts. In a high frequency AC-coupling model the stray capacitance of the series resistors $R_{1}+R_{2}$ and $R_{3}+R_{4}$ are parallel to the APD's capacitance and therefore directly add up to the input capacitance of the detector. The choice of using two resistors instead of one in the design phase was done to effectively half this stray capacitance even further.

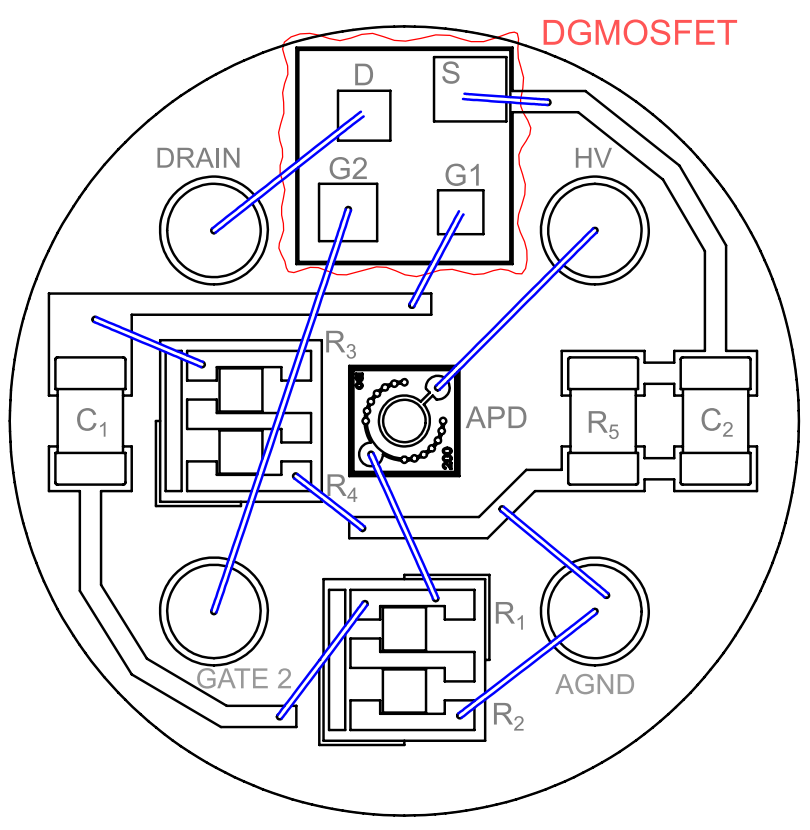

Fig. 3. Bonding diagram featuring no crossings of bonding wires (with courtesy of Laser Components)

The bonding diagram as in Fig. 3 shows the wirebonds (blue), the transistor (red), the passive parts, the APD die (in center) as well as the pins of the TO-46 case. The resulting detector is shown in Fig. 4. It must be noted, that the diameter of the hybrid package of $5.4 \mathrm{~mm}$ is the same size as previously of the APD alone.

As this paper intends to address only the differences in relation to hybrid and non-hybrid, preemptive measures were taken. First the APD dies used in the discrete detector as well as in the hybrid detector stem from the same wafer, thus the variance in electrical characteristics caused by tolerances is minimized. Second the detector overall PCBs were essentially the same for the hybrid detector as well as for the discrete detector. Only the parts, which moved from the discrete circuit into the hybrid detector were removed and the PCB remained unchanged. 


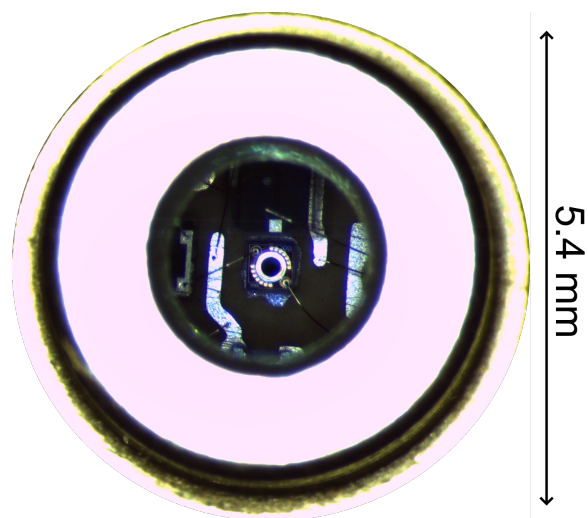

Fig. 4. Hybrid detector showing the APD and some of the wirebonds through a flat BK7 window glass (with courtesy of Laser Components)

\section{Measurements and Results}

There were mainly four measurements/experiments carried out in a laboratory environment to compare the hybridized against the discrete detector:

- Input capacitance of detector

- Detector SNR (without background noise)

- Noise equivalent power (NEP)

- EMI immunity (only qualitatively)

\section{A. Measurement Setup}

A reproducible measurement setup as depicted in Fig. 5 was designed. This setup supports different measurements like input capacitance estimation or signal-to-noise with no outside background noise photons present. It consists of a laser source $(\lambda=1535 \mathrm{~nm})$ with approximately $4 \mathrm{~ns}$ pulse width, a neutral density filter wheel with a position scale (to attenuate the laser light), an integrating sphere, and an APD case compatible light tube. The detector board can be mounted directly, fitting the entire APD case without remaining spacing into the light tube.

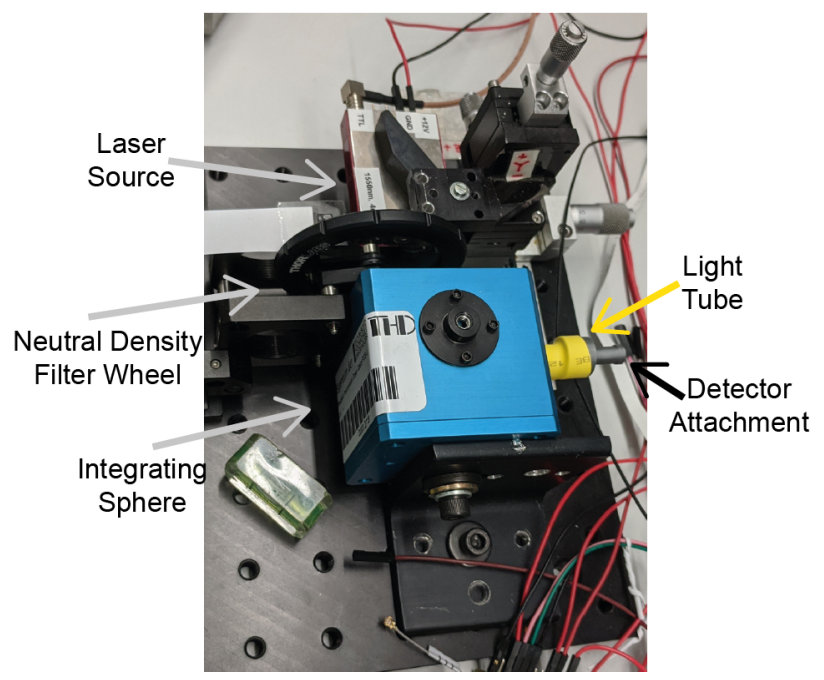

Fig. 5. Measurement setup in the laboratory based on an integrating sphere to evaluate and compare detector designs

\section{B. Estimation of Input Capacitance}

The capacitance of the APD in the TO-46 case. was measured to be $1.7 \mathrm{pF}$ beforehand using a capacitance measurement setup [13, p. 162].

The expected combined reduction of the detector input capacitance can be estimated at $0.6-0.8 \mathrm{pF}$. The sources for the reductions are:

- $0.5 \mathrm{pF}$ from the TO-46 casing (according to internal data of Laser Components DG, Inc.)

- $0.1 \mathrm{pF}$ from the transistor package (see [12])

- up to $0.2 \mathrm{pF}$ from the PCB traces, soldering joints and wire-bondable resistors

To estimate the reduction of input capacitance of the detector a measurement using a low capacitance probe, LeCroy AP020, directly at the drain of the transistor in conjunction with the setup as seen in Fig. 5 was conducted. In addition to the measurement a LTspice simulation (Fig. 6) to correlate experiment and theory was carried out. In the simulation circuit, the detectors input capacitance reduction is fully attributed to reducing the APD's capacitance. For the measurement the laser was triggered and the resulting amplified laser pulse signal in form of a fast ramp-down and slow ramp-up (Fig. 7) was recorded. This signal shape is strongly impacted by the input capacitance of the detector.

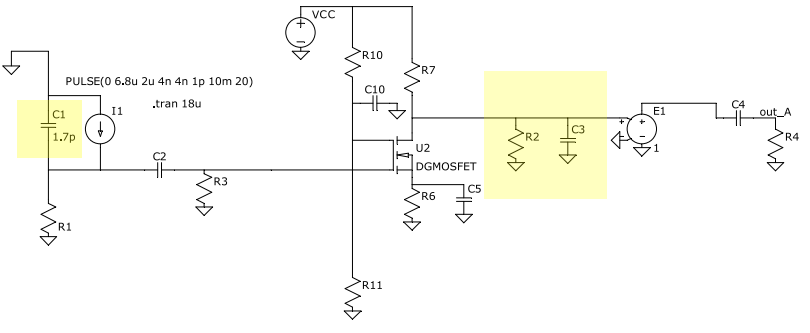

Fig. 6. The LTspice simulation circuit was used for estimating the change of input capacitance. Therein $\mathrm{C} 1$ represents the APD capacitance and R2/C3 represent the AP020 probe.

(a)
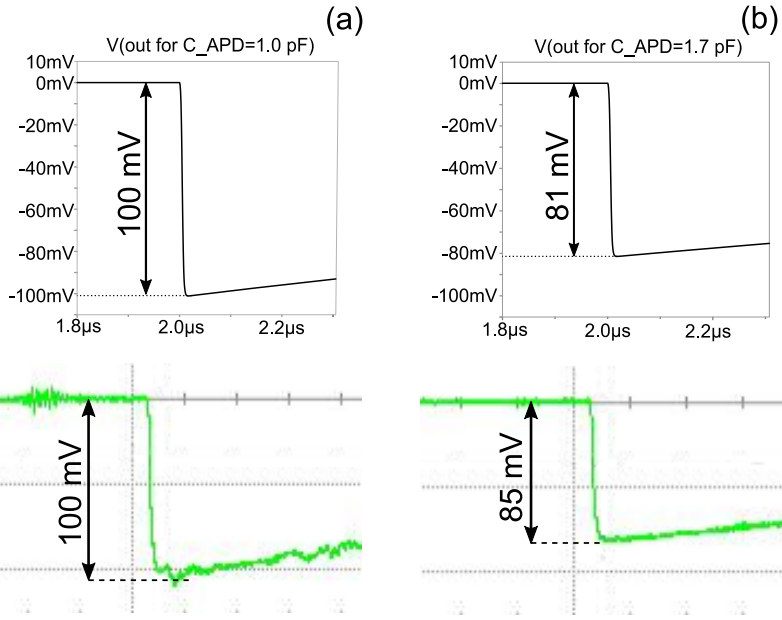

(b)

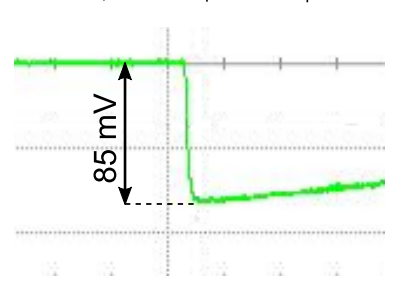

Fig. 7. The top graphs show the LTspice simulated signals at the drain pin. The bottom graphs show measured signals using the AP020 probe and an oscilloscope. The reduction in capacitance, which leads to a higher input bandwidth, causes the difference of signal height. Column (a) denotes the hybrid, (b) the non-hybrid detector. 
The simulation result matches the measured data well and therefore a reduction of the capacitance of $0.7 \mathrm{pF}$ can be assumed.

\section{Detector SNR}

To measure the SNR of the detector without background noise, the measurement setup as depicted in Fig. 5 was used. The position of the filter wheel was chosen such, that the detector is still operating in linear mode and without clipping. This position was then fixed for all subsequent SNR measurements. The APD was operated at a bias voltage of $56 \mathrm{~V}$, which is according to the manufacturing data sheet, the voltage which leads to the best SNR of the APD itself.

Figure 8 shows both the pulse, the maximum peak signal level, the noise, and its respective RMS level for respectively one time series each.
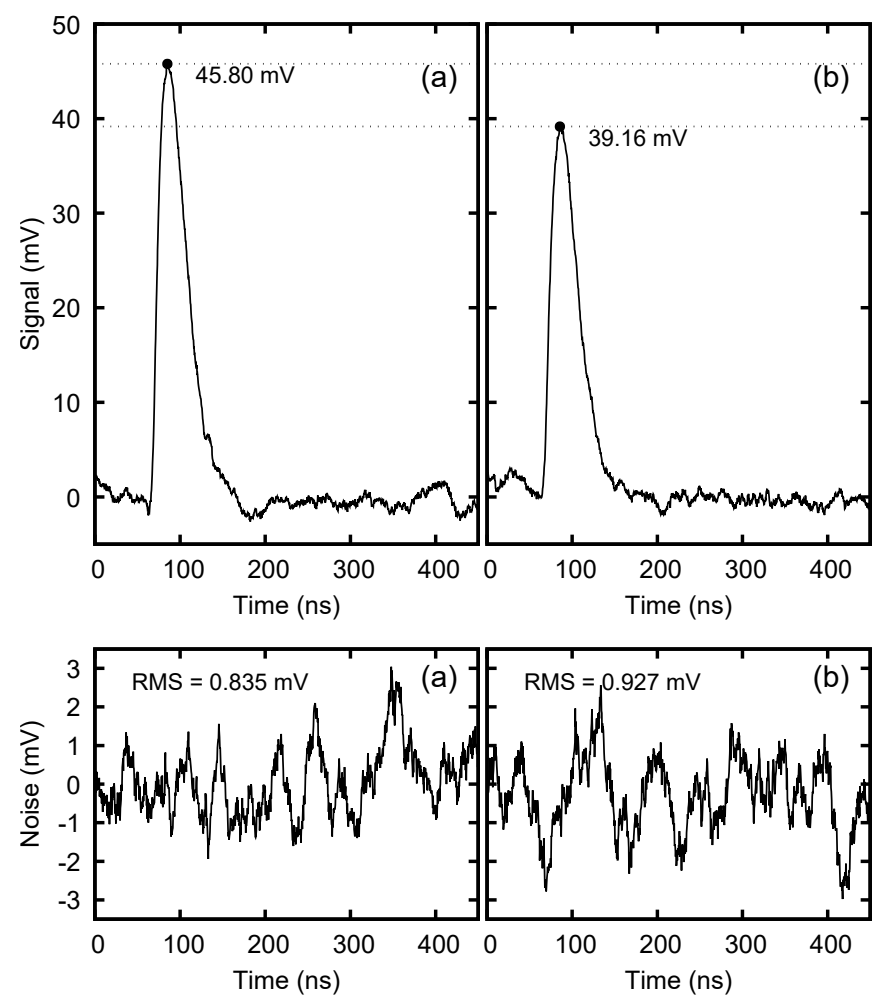

Fig. 8. (a) hybrid detector, (b) non-hybrid detector

Top figure: Detected laser pulse, Bottom figure: Noise with laser turned off

\begin{tabular}{|l|c|c|}
\hline & Hybrid & Non-Hybrid \\
\hline FWHM pulse response & $38.1 \mathrm{~ns} \pm 0.8 \mathrm{~ns}$ & $38.8 \mathrm{~ns} \pm 1.1 \mathrm{~ns}$ \\
\hline RMS noise & $810 \mu \mathrm{V} \pm 56 \mu \mathrm{V}$ & $875 \mu \mathrm{V} \pm 58 \mu \mathrm{V}$ \\
\hline Signal peak & $49.2 \mathrm{mV} \pm 1.9 \mathrm{mV}$ & $40.0 \mathrm{mV} \pm 1.8 \mathrm{mV}$ \\
\hline SNR & $60.7 \pm 6.5$ & $45.7 \pm 5.1$ \\
\hline Number of Traces & 850 & 760 \\
\hline
\end{tabular}

TABLE I

MEASUREMENT RESULTS FOR MULTIPLE TRACES

To obtain the SNR, the signal peak level is being divided by the RMS noise level. The results of these measurements are summarized in Table I. In this measurement, the improvement in SNR is 32 percent.

To further analyze the influence of varying APD bias voltage, a measurement series involving a sweep in the range from 50 to $60 \mathrm{~V}$ has been conducted.

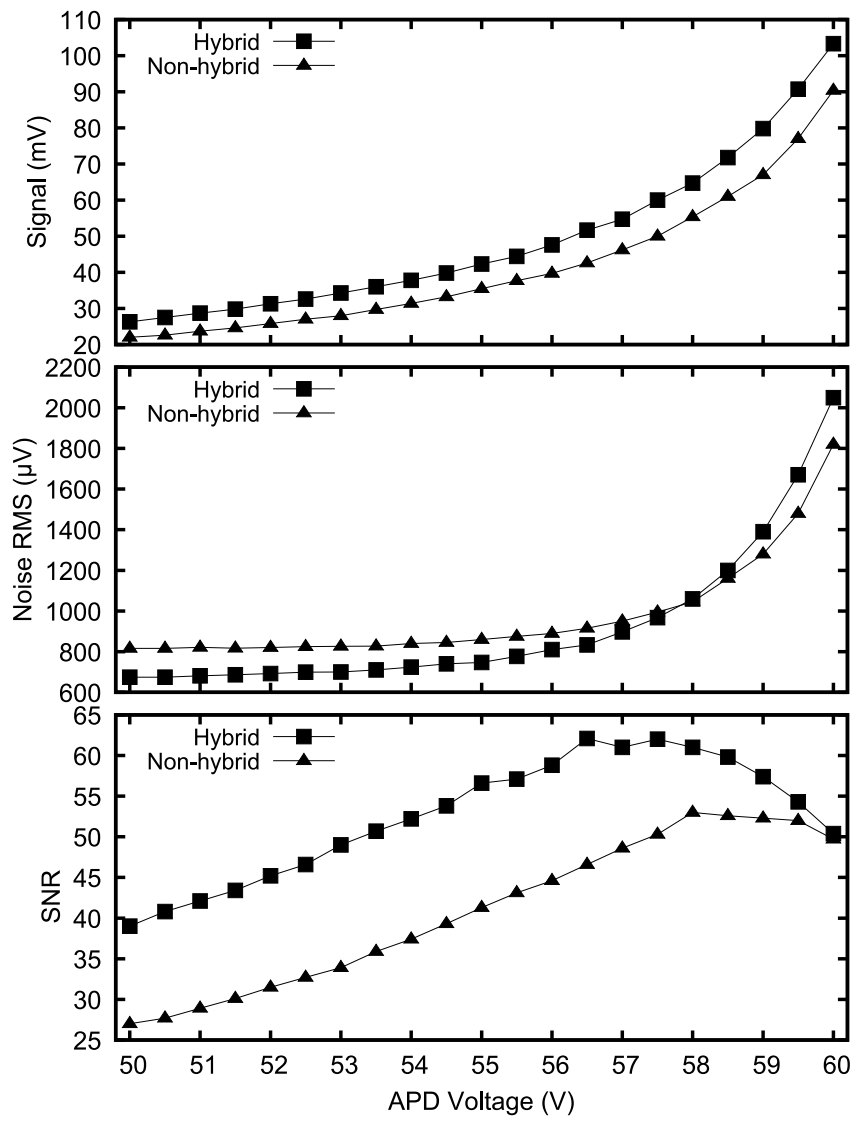

Fig. 9. Measurement series of signal peak, noise RMS and SNR over APD high voltage sweep

For low APD voltages in the range from 50 to $55 \mathrm{~V}$, most of the noise part stems from the transistor's and bias circuit's added noise. One can clearly see, that the hybrid detector has a lower integrated noise than the discrete detector (Fig. 9). This seems to be counter-intuitive as the expectation is, that a lower input capacitance leads to a higher noise bandwidth and therefore to a higher integrated noise voltage. The lower integrated noise can be explained by looking at the voltage noise densities of the detectors as in Fig. 10. Simulations show, that the first region of interest $(0.1$ to $2 \mathrm{MHz})$ is mainly influenced by the input capacitance of the detector. As expected the hybrid detector therein shows a higher noise floor than the discrete detector. The second region of interest ( 2 to $10 \mathrm{MHz}$ ) on the contrary shows a lower noise peak for the hybrid than the non-hybrid detector. This difference is causing the integrated noise to be lower for the hybrid design. Simulations show, that the peak in this region can be attributed to a parasitic capacitive coupling between the input of the detector and the output of the equalizer, which overall form a non-inverting topology (positive feedback). The fact, that the first amplification stage and the APD are in the same case, yields a shielding effect, which reduces 


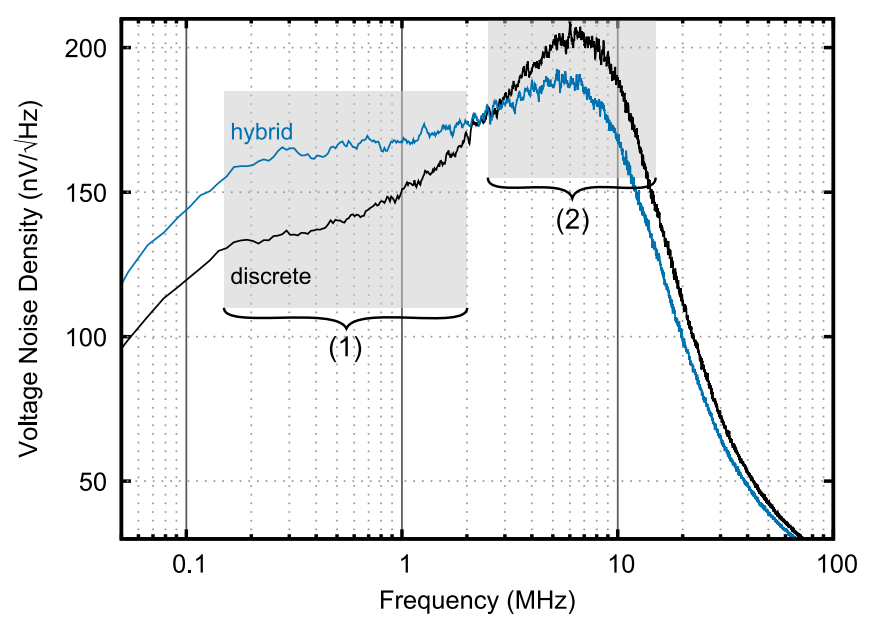

Fig. 10. Comparison of voltage noise density at 56 V APD bias, (blue) hybrid, (black) discrete detector, with regions of interest (1) due to input capacitance and (2) due to parasitic feedback capacitance

this parasitic capacitance coupling. For frequencies above 10 $\mathrm{MHz}$, the roll-off is mainly formed by the equalizer. The combination of these two factors lead to a lower integrated noise voltage for the hybrid detector in the bias voltage range below $58 \mathrm{~V}$. Starting at around $58 \mathrm{~V}$, the hybrid detector shows a higher integrated noise voltage as the non-hybrid. For bias voltages above $58 \mathrm{~V}$ the dark current of the APD and the associated current shot noise further increases. The effect of the lower input capacitance and the attributed higher noise bandwidth now dominate the effect of reducing the parasitic capacitance and therefore the hybrid detector shows higher integrated noise for higher bias voltages closer to breakdown. For the signal peak, the hybrid detector shows an increase in level of approximately 10 percent throughout the full range. This can be attributed to the reduced input capacitance. The signal to noise ratio peaks at around $56.5 \mathrm{~V}$ for the hybrid detector, while it peaks at around $58.5 \mathrm{~V}$ for the discrete detector. At the APDs optimum bias voltage level $(56 \mathrm{~V})$, the improvement in SNR is 32 percent. For lower APD voltages, this even increases to 45 percent, while at higher APD voltages approaching the APD breakdown voltage, the improvement will start to disappear completely (starting at about $59 \mathrm{~V}$ ).

\section{Noise Equivalent Power}

The noise equivalent power and input referred current noise density of both detectors was calculated using the Thorlabs whitepaper [14]. In order to do so, the measured numbers of our amplifiers were converted to equivalent transimpedance amplifiers. The frequency range is $40 \mathrm{kHz}-10 \mathrm{MHz}$. The results are shown in Table II.

\section{E. EMI immunity}

The expectation is, that the integration of the high impedance parts into the same casing yields clear shielding benefits. Therefore a qualitative measurement using a different laser, which deliberately emits high electromagnetic interference, was conducted. In this experiment, the filter wheel was

\begin{tabular}{|l|c|c|}
\hline & Hybrid & Non-Hybrid \\
\hline Noise equivalent power & $36 \frac{\mathrm{fW}}{\sqrt{\mathrm{Hz}}}$ & $50 \frac{\mathrm{fW}}{\sqrt{\mathrm{Hz}}}$ \\
\hline Input referred noise & $0.39 \frac{\mathrm{pA}}{\sqrt{\mathrm{Hz}}}$ & $0.54 \frac{\mathrm{pA}}{\sqrt{\mathrm{Hz}}}$ \\
\hline Equivalent transimpedance & $686 \mathrm{k} \Omega$ & $562 \mathrm{k} \Omega$ \\
\hline Bandwidth & $10 \mathrm{MHz}$ & $10 \mathrm{MHz}$ \\
\hline
\end{tabular}

TABLE II

COMPARISON OF NOISE PARAMETERS

adjusted between both measurements in a way, such that the signal shapes can be referenced to the same maximum level.

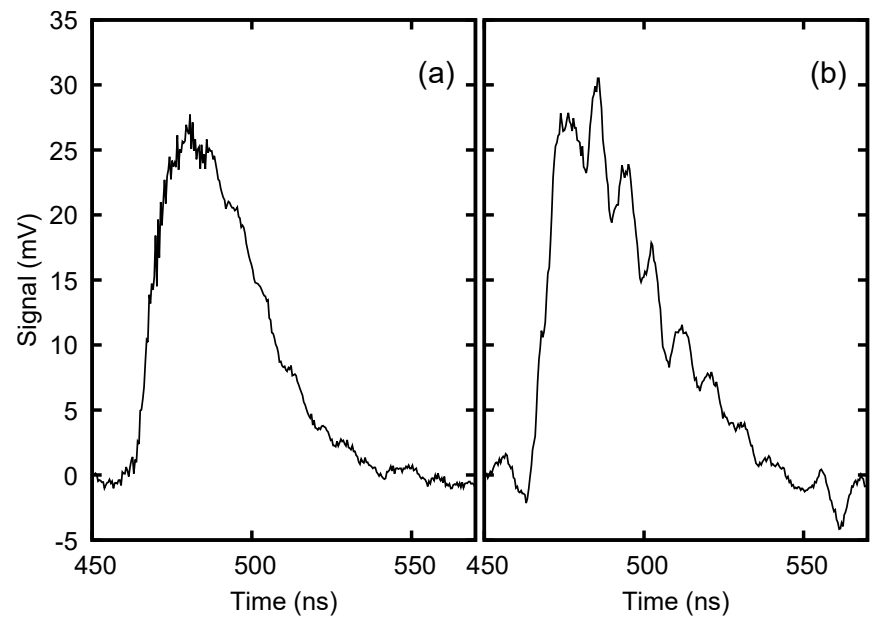

Fig. 11. Electromagnetic interferences, (a) hybrid, (b) non-hybrid

The graph as in Fig. 11 shows a smoother pulse curve for the hybrid detector and thus the improved performance with regard to electromagnetic interference caused by the laser.

\section{F. Comparison to State of the Art}

This hybrid design is compared against three state of the art integrated detectors [7] [8] [9] and two commercially available hybrid detectors [15] [16]. Table III shows the comparison data. This work is the only detector in this comparison, which uses a voltage mode amplifier as first stage. It must be noted, that this newly introduced detector has exceptional low input referred current noise and high transimpedance. It must be noted though, that the bandwidth of $10 \mathrm{MHz}$ is low compared to other detectors. This leads to a very high sensitivity. The gain control varies between different detectors. This detectors high dynamic range $(120 \mathrm{~dB})$ is achieved by controlling the APD bias voltage ( $=87 \mathrm{~dB}$ dynamic range) and the voltage at Gate2 (= $33 \mathrm{~dB}$ dynamic range). In the applied system, digital processing is used to detect signal clipping and automatically regulate these two voltages.

\section{SYSTEM INTEGRATION}

The system in Fig. 12 consists of a pair of binoculars, a laser, a detector and additional processing electronics. The main key parameters of the system are grouped together in Table IV. The system is classified as eye safe class 1 DIN 


\begin{tabular}{|c|c|c|c|c|c|c|}
\hline & This Work & {$[7]$} & {$[8]$} & {$[9]$} & {$[15]$} & {$[16]$} \\
\hline Type & Hybrid & Integrated & Integrated & Integrated & Hybrid & Hybrid \\
\hline PD Type & InGaAs APD & InGaAs PIN & InGaAs APD & Si APD & InGaAs APD & InGaAs APD \\
\hline PD Wavelength $(\mathrm{nm})$ & 1535 & 1550 & 1550 & 905 & 1550 & 1550 \\
\hline Amplifier Configuration & Voltage Pre-Amplifier & TIA & TIA & TIA & TIA & TIA \\
\hline Laser pulse width $(\mathrm{ns})$ & 4 & 4 & 5 & 5 & N/A & N/A \\
\hline Bandwidth $(\mathrm{MHz})$ & 10 & 720 & 160 & 153 & 50 & 100 \\
\hline Input Referred Noise $(\mathrm{pA} / \sqrt{\mathrm{Hz}})$ & 0.39 & 6.3 & 1.36 & 0.89 & 0.67 & 2.0 \\
\hline TZ Gain $(\mathrm{dB} \Omega)$ & 116 & 76.3 & 110 & 106 & 96 & 94 \\
\hline Gain Control & External voltage & Automatic & Dual Gain & Programmable & N/A & N/A \\
\hline Dynamic Range & $1: 100000$ & $1: 11760$ & $1: 12000$ & $1: 10000$ & N/A & N/A \\
\hline
\end{tabular}

TABLE III

Performance Comparison of Detectors

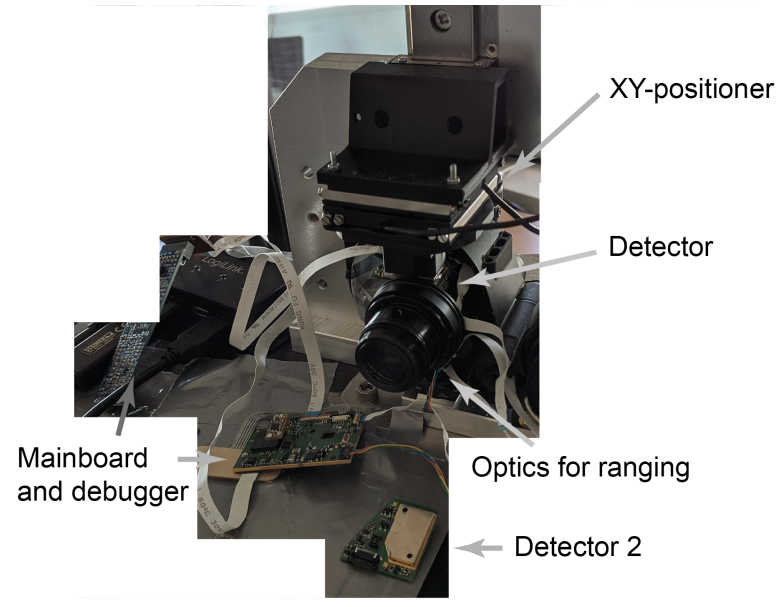

Fig. 12. Detector applied in Steiner binoculars with courtesy of company Steiner Optik GmbH

EN 60825-1/11.01. There is also a system to box the detector into the signal maximum accurately using nano-positioners and mechanical support in a closed loop setup. In order to minimize influence of weather fluctuations, measurements and signal boxing were repeated many times in order to generate the results as shown in Table V.

For the measurements, three main targets as shown in Fig. 13 were used. The first target shows a forest, which usually is a challenging target because of its low albedo and high surface non-uniformity. The second target shows a house, which is located at $1.6 \mathrm{~km}$ distance and represents an easy to measure target. The measurements on this target were worsened by a neutral density filter in front of the laser. Target three shows a house at $11.3 \mathrm{~km}$ distance. The house has a small treeline in front of its wall.

At the time series (Fig. 14), one can see that the peak at the $11.3 \mathrm{~km}$ mark is clearly distinguishable from the noise and can easily be detected by the processing algorithms later on.

The measurement results for the given targets (Table V) show, that at conditions with strong daylight (i.e. high shot noise component from background), the hybrid detector's improvement gets smaller ( 3 and 10 percent). When the daylight is bandpass filtered, this changes to an improvement of 8 and 21 percent respectively. This confirms, that the decreased input

\begin{tabular}{|l|c|}
\hline Laser pulse FWHM & $4 \mathrm{~ns}$ \\
\hline Laser pulse energy & $40 \mu \mathrm{J}$ \\
\hline Laser beam full angle divergence & $0.4 \mathrm{mrad}$ \\
\hline Laser $\mathrm{M}^{2}$ & 1.3 \\
\hline Repetition rate & $100 \mathrm{~Hz}$ \\
\hline Optical wavelength & $1535 \mathrm{~nm}$ \\
\hline Clear optical aperture & $30 \mathrm{~mm}$ \\
\hline Receive path efficiency & $54 \%$ \\
\hline Transmission path efficiency & $100 \%$ \\
\hline APD gain & 10 \\
\hline Quantum efficiency of APD & $85 \%$ \\
\hline Optical bandwidth & $630 \mathrm{~nm}$ \\
\hline Dark current & $10 \mathrm{nA}$ \\
\hline Ionization factor & 0.2 \\
\hline Noise excess factor & 3.52 \\
\hline Diameter of APD active area & $200 \mu \mathrm{m}$ \\
\hline Default measurement time & $400 \mathrm{~ms}$ \\
\hline
\end{tabular}

TABLE IV

KEY PARAMETERS OF LASER RANGE FINDING SYSTEM

\begin{tabular}{|c|c|c|c|c|c|}
\hline Target & daylight & visibility & S/N hybrid & S/N discrete & diff \\
\hline 1 & strong & $>25 \mathrm{~km}$ & 7.94 & 7.23 & $10 \%$ \\
\hline 2 & strong & $>25 \mathrm{~km}$ & 9.37 & 9.13 & $3 \%$ \\
\hline 1 & BP filtered & $>25 \mathrm{~km}$ & 9.53 & 7.87 & $21 \%$ \\
\hline 2 & BP filtered & $>25 \mathrm{~km}$ & 13.19 & 12.18 & $8 \%$ \\
\hline 1 & weak & $<5 \mathrm{~km}$ & 7.91 & 6.21 & $27 \%$ \\
\hline 3 & weak & $<5 \mathrm{~km}$ & 7.8 & 6.14 & $27 \%$ \\
\hline 1 & weak & $<15 \mathrm{~km}$ & 26.33 & 21.95 & $20 \%$ \\
\hline
\end{tabular}

TABLE V

MEASUREMENT RESULTS FOR GIVEN TARGETS

capacitance, leads to an increase of noise bandwidth of the detector. At weak daylight conditions the hybrid detector has an advantage of up to 27 percent over the discrete detector.

\section{CONCLUSION}

This work presented the integration of an InGaAs APD with the first amplification stage incorporated in the APD case. The results show a significant reduction of input capacitance of 0.7 $\mathrm{pF}$ and improved immunity against electromagnetic interfer- 


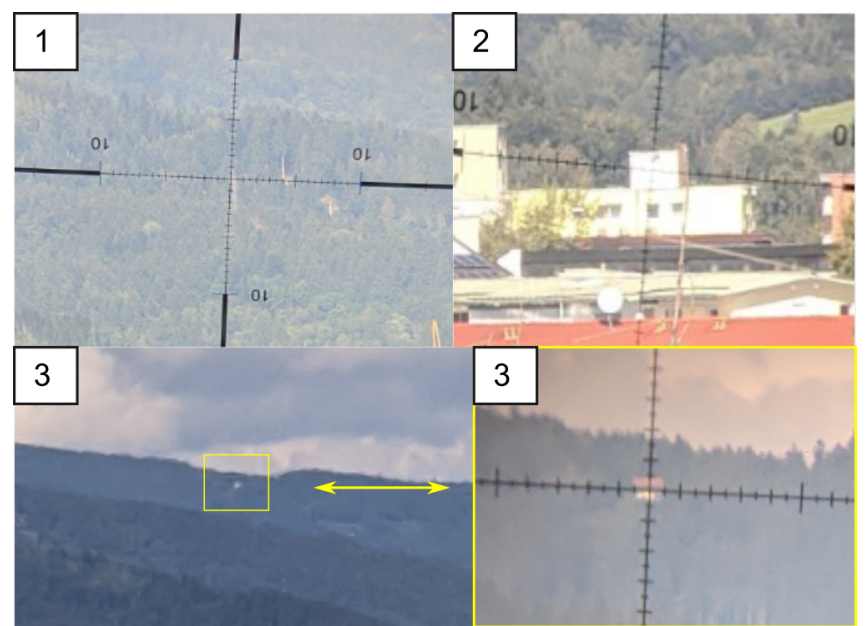

Fig. 13. Targets, (1) tree in forest at $6.3 \mathrm{~km},(2)$ house at $1.6 \mathrm{~km},(3)$ house at $11.3 \mathrm{~km}$ distance (zoomed in)

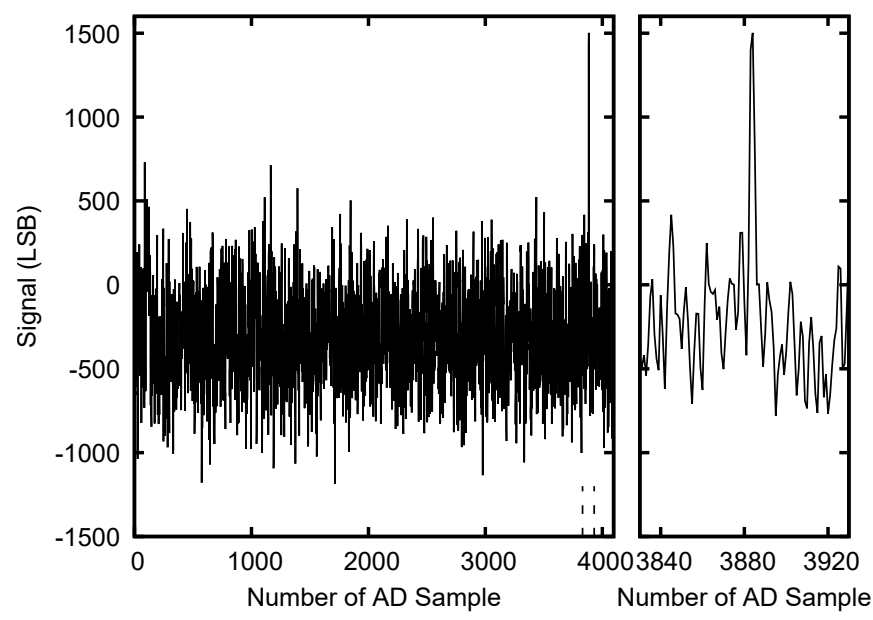

Fig. 14. Exemplary digitized time series of a $11.3 \mathrm{~km}$ measurement on target 3 using 40 pulses (i.e. $0.4 \mathrm{~s}$ measurement time) showing a SNR of 5 . This peak can be detected by the detection algorithm with high confidence.

ence when the hybrid detector is compared to the equivalent discrete detector. There was also an additional reduction of parasitic input-output coupling of the detector. This allowed the hybrid design to have lower total integrated noise voltage, while having a higher noise and signal bandwidth, when compared to the discrete design. A very low noise equivalent power of $36 \mathrm{fW} / \sqrt{\mathrm{Hz}}$, while providing an equivalent transimpedance gain of $686 \mathrm{k} \Omega$ was achieved. This allows an eye safe laser ranging system using the presented hybrid detector to surpass the $10 \mathrm{~km}$ range barrier with high confidence. The hybrid detector is superior to the discrete detector in every measured scenario, even at very strong daylight conditions.

\section{ACKNOWLEDGMENT}

The authors are grateful to Gerd Lottes and Oliver Jäschke from company Steiner Optik GmbH for funding the project, Mike Hodges and Dragan Grubisic from company Laser Components $\mathrm{GmbH}$ for building first prototypes of the hybrid detector and Prof. Raimund Förg from Deggendorf Institute of Technology for general support.

\section{REFERENCES}

[1] A. A.S., R. K.S., and B. J., "Object-based land cover classification using airborne LiDAR," Remote Sensing of Environment, vol. 112, no. 6, pp. 2988-2998, 2008, doi: 10.1016/j.rse.2008.02.004.

[2] L. Combrinck, "Satellite Laser Ranging," In: Xu G. (eds) Sciences of Geodesy - I. Springer, Berlin, Heidelberg, 2010, doi:

10.1007/978-3-642-11741-1_9.

[3] A. Markus-Christian, M. B. Thierry, L. Marc, A. M. Risto, and R. Marc, "Laser ranging: a critical review of usual techniques for distance measurement," Optical Engineering, vol. 40, no. 1, pp. 10-19, 2001, doi: $10.1117 / 1.1330700$.

[4] H. T. Friis, "Noise Figures of Radio Receivers," Proceedings of the IRE, vol. 32, no. 7, pp. 419-422, July 1944, doi: 10.1109/JRPROC.1944.232049.

[5] A. A. Abidi, "On the operation of cascode gain stages," IEEE Journal of Solid-State Circuits, vol. 23, no. 6, pp. 1434-1437, Dec. 1988, doi: 10.1109/4.90043.

[6] A. Baharmast, J. Kostamovaara, "High-speed wide dynamic range linear mode time-of-flight receiver based on zero-crossing timing detection," Opt. Eng. 59(10) 104102 (14 October 2020), doi: 10.1117/1.OE.59.10.104102

[7] C. Hong, S. Kim, J. Kim and S. M. Park, "A Linear-Mode LiDAR Sensor Using a Multi-Channel CMOS Transimpedance Amplifier Array," IEEE Sensors Journal, vol. 18, no. 17, pp. 7032-7040, 1 Sept.1, 2018, doi: 10.1109/JSEN.2018.2852794.

[8] H. Cho, C. Kim and S. Lee, "A High-Sensitivity and Low-Walk Error LADAR Receiver for Military Application," in IEEE Transactions on Circuits and Systems I: Regular Papers, vol. 61, no. 10, pp. 3007-3015, Oct. 2014, doi: 10.1109/TCSI.2014.2327282.

[9] H. Zheng, R. Ma, M. Liu and Z. Zhu, "A Linear Dynamic Range Receiver With Timing Discrimination for Pulsed TOF Imaging LADAR Application," IEEE Transactions on Instrumentation and Measurement, vol. 67, no. 11, pp. 2684-2691, Nov. 2018, doi: 10.1109/TIM.2018.2826860.

[10] T. Muoi, "Receiver design for high-speed optical-fiber systems," Journal of Lightwave Technology, vol. 2, no. 3, pp. 243-267, 1984, doi: 10.1109/JLT.1984.1073617.

[11] K. Ogawa, "Considerations for Optical Receiver Design," IEEE Journal on Selected Areas in Communications, vol. 1, no. 3, pp. 524--532, 1983, doi: 10.1109/JSAC.1983.1145939.

[12] Texas Instruments, "AN-1205 Electrical Performance of Packages," May. 2004. [Online]. Available: https://www.ti.com/lit/an/snoa405a/snoa405a.pdf

[13] Larry K. Baxter, Capacitive Sensors: Design and Applications. Piscataway: IEEE Press, 1997.

[14] V. Mackowiak, J. Peupelmann, Y. Ma, A. Gorges, "NEP - Noise Equivalent Power," Thorlabs Inc., Thorlabs GmbH. Accessed: Dec. 28 2020. [Online]. Available: https://www.thorlabs.com/ images/TabImages/Noise_Equivalent_Power_White_Paper.pdf

[15] Product Datasheet C30659-1550-R2AH, Excelitas Technologies Corp.

[16] Product Datasheet IAG200H2, Laser Components USA, Inc.

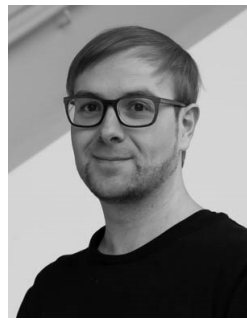

Michael Kufner was born in Deggendorf, Germany in 1987. He received the B.E. and M.Eng. degrees in electrical engineering and information technology from the Deggendorf Institute of Technology, Germany in 2011 and 2012, respectively.

Since 2012 he has been with the optical engineering group at the Deggendorf Institute of Technology, where he mainly does research on laser range finding for hand-held devices. His primary interests include high-sensitivity optical receivers and optimizing the pulse processing algorithms and systems on these devices.

$\mathrm{He}$ is currently pursuing a doctoral degree at the University of Stuttgart, Faculty for Aerospace Engineering and Geodesy, Pfaffenwaldring 27, 70569 Stuttgart, Germany. 


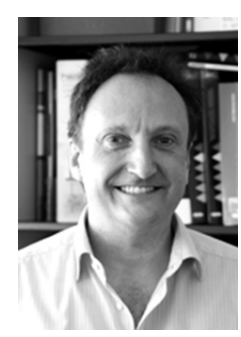

Josef Kölbl was born in Passau, Germany in 1966. He studied electrical engineering at Fachhochschule Regensburg. Currently he holds a $\mathrm{PhD}$ from University of Central Lancashire in England. Since 2010 he has a professorship at Technische Hochschule Deggendorf, working in the fields of electronics and laser ranging.

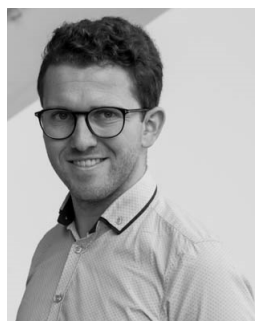

Raphael Lukas was born in Bogen, Germany in 1993. He received the B.E. degree in electrical engineering from the Deggendorf Institute of Technology, Germany in 2020.

Since 2012 he has been with the optical engineering group at the Deggendorf Institute of Technology, where he mainly does research on laser range finding for hand-held devices. His primary interests include high-sensitivity receivers and optimizing hardware layouts and circuits for these devices.

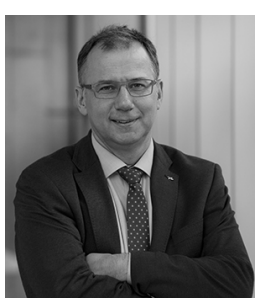

Thomas Dekorsy graduated from the RWTH Aachen, Germany in the field of femtosecond spectroscopy. He worked as a division head at the Helmholtz-Center Dresden-Rossendorf, Germany with a THz-free-electron laser until 2004. He became full professor at Konstanz University, Germany in 2005. In 2016, he became Director of the Institute for Technical Physics of the German Aerospace Center (DLR) in Stuttgart, Germany and is Professor at Stuttgart University, Germany in the Faculty of Aerospace Engineering and Geodesy. He published more than 200 research papers and holds 5 patents in the field of lasers and optoelectronic devices. 